\title{
GENETIC AND ENVIRONMENTAL EFFECTS ON SELF-REPORTED DEPRESSIVE SYMPTOMS IN A GENERAL POPULATION TWIN SAMPLE
}

\author{
J. L. Silberg, ${ }^{* \dagger}$ A. C. Heath, $\ddagger$ R. Kessler, $\S$ M. C. Neale, ${ }^{*} \uparrow$ J. M. Meyer, ${ }^{*}$ \\ L. J. Eaves* $\|$ and K. S. KendLER* $\|$
}

*Department of Human Genetics, Medical College of Virginia, Box 33, Richmond, VA 23298-0033, U.S.A.; $\Varangle$ Department of Psychiatry, Washington University Medical School, 4940 Audubon Avenue, St. Louis, MO 63110 , U.S.A.; \$Institute For Social Research, University of Michigan, Box 1248, Ann Arbor, MI 48106-1248, U.S.A.; I Department of Pyschology, Virginia Commonwealth University, Richmond, VA 23298, U.S.A. and \|Department of Psychiatry, Medical College of Virginia, Box 710, Richmond, VA 23298, U.S.A.

(Received 24 May 1989; revised 2 October 1989)

\begin{abstract}
Summary-To determine the etiology of self-reported depressive symptoms and their co-occurrence in the general population, multivariate genetic models were fitted to the responses of 771 female twin pairs $(463 \mathrm{MZ}, 308 \mathrm{DZ})$ to a 20 -item epidemiological depression inventory (CES-D scale). A model which contained one common genetic factor, one shared environmental factor, and four unique environmental factors provided a useful account of symptom covariation. Under this model, the four non-shared environmental factors explained the largest proportion of variance in response to the CES-D scale, whereas a single common genetic factor explained substantially less of the variation in symptomatology. Consistent with previous findings (Kendler, Heath, Martin, \& EAves, Archives of General Psychiatry 43, 213-221, 1986) shared environmental influences were found to play a relatively minor role in the report of depressive symptoms. These results suggest that while genetic factors do contribute to the covariation among symptoms of depression, it is the largely non-shared environmental factors that account for the co-occurrence of symptoms in the general population.
\end{abstract}

\section{INTRODUCTION}

EVIDENCE from numerous twin, family, and adoption studies has demonstrated the importance of both genetic and environmental factors in affective illness. In a review of the twin literature on affective disorders GERSHON, BUNNEY, LECKMAN, VAN EERDEWEGH, and DeBAUCHE (1976) reported an overall concordance rate of $69.2 \%$ for monozygotic twins and $13.3 \%$ for dyzygotic twin pairs, providing support for a genetic predisposition to this disorder.

Genetic effects have been relatively well established in bipolar depression (WINOKUR \& Clayton, 1967; Bertelsen, Harvald, \& Hauge, 1977; Mendlewicz \& Rainer, 1977). However, a genetic susceptibility to unipolar depression, the more common form of affective illness, has not been unequivocably demonstrated. Genetic transmission of unipolar depression has been found in a number of twin (BERTELSEN et al., 1977; TORGERSEN, 1986) and adoption studies (CADORET, 1978; KETY, 1985; WENDER et al., 1986). However, other studies have shown similarity among relatives for unipolar depression to be predominantly environmental in origin (Von KNorRING, Cloninger, BoHman, \& Sigvardson, 1983; Cadoret, O'Gorman, Heywood, \& Troughton, 1985).

†To whom correspondence should be addressed. 
Inconsistencies among genetic studies of unipolar depression have been associated with a number of factors, including methodological variability, heterogeneity of affective illness and the use of varying criteria in diagnosing depression (TORGERSEN, 1986). Lack of agreement in diagnostic criteria is especially problematic for deriving reliable estimates of genetic and environmental components of variance. Since symptoms comprising an observable depressive syndrome or phenotype are likely to have unequal heritabilities (Kendler, Heath, Martin, \& Eaves, 1987), definitions based upon different subsamples of symptoms are likely to result in divergent estimates of heritability.

To avoid the problem of unreliable diagnosis and obtain a more representative sample of depressed individuals, several investigators have studied genetic effects over the entire range of depressive symptoms using data from general population samples. JARDINE, MARTIN, \& HENDERSon (1984) found that variation in symptoms of anxiety and depression within a large Australian twin sample was due almost entirely to additive genetic effects and those environmental experiences unique to the individual. While JARDINE $e t$ al. studied the genetic and environmental effects on total scale scores of anxiety and depressive symptoms, KENDLER et al. (1986) examined the sources of variation in the 14 separate anxiety and depression symptoms. Their results convincingly demonstrated the importance of unique environmental and genetic influences in explaining variation in most of these 14 symptoms. Those experiences shared by both members of a twin pair, including parenting style and early social and familial experiences, previously considered to be of etiological significance in later onset of psychiatric symptomatology (Brown, Harris, \& CoPEland, 1977; PARKER, 1979), were found to play a relatively insignificant role in many of these symptoms. In a later analysis, KendLER et al. (1987) examined the covariation of the above symptoms of anxiety and depression. Using multivariate genetic models developed by MARTIN \& EAVES (1977), KENDLER et al. (1987) investigated whether the same genetic and/or environmental factors that influenced one symptom were responsible for explaining variation in other related symptoms. I' Iheir results revealed a non-specific common genetic factor that influenced an overall susceptibility to psychiatric distress, and found that it was the environment, specifically those environmental factors affecting only one member of the twin pair, that determined whether this genetic liability was expressed as symptoms of anxiety or symptoms of depression.

The combination of using a general population sample within a conventional twin design is a powerful way of resolving genetic and environmental contributions to symptom variation while avoiding the biases inherent in studying clinically based samples and their families. This method also permits the use of multivariate genetic models to examine those factors responsible for the patterning of symptoms. In the present study, we wish to further examine the effect of genes and environment on the variation in self-reported depressive symptoms in a new general population sample of adult twins, using a 20-item epidemiological depression inventory (RADLOFF, 1977). Specifically, we use multivariate genetic models (c.f. KENDLER et al., 1987) to explore the etiology of symptom covariation by determining whether it is common genetic and/or common environmental factors that are most influential in determining the pattern of covariation among individual symptoms of depression. 
METHODS

\section{Sample}

Subjects were a subsample of 771 adult female caucasian twin pairs (463 MZ, $308 \mathrm{DZ}$ pairs) selected from a large epidemiological study of anxiety and depression. Between December 1986 and April 1988 questionnaires were mailed to 3,642 female caucasian twins from the Virginia Twin Register (CoRey, Eaves, Mellen, \& NANCE, 1986) in which at least one member of a pair had responded to a previous twin questionnaire. Of the same-sex female twins in the sample, 2,146 individual twins received and completed the questionnaire (a $62 \%$ individual response rate); 771 complete twin pairs (1,542 individuals) had zygosity information available and were used in the data analysis. These women ranged in age from 18 to 52 years, with a mean of 31.2 and a standard deviation of 8 years. The data collection was confined to females because of the higher rate of psychiatric distress in females and the difficulty involved in obtaining a sufficiently large sample of males (or non-whites) for the analysis of sex (race) specific effects. Zygosity determination was made by the responses of twins to several questions regarding physical similarity and the extent to which one twin was mistaken for another. Such methods have been found to show good agreement with zygosity determination through more rigorous blood typing procedures (NICHOLs \& BILBRO, 1966).

\section{Instruments}

The Epidemiology of Anxiety and Depression project is comprised of two waves of data collection. In the first wave, twins are mailed a questionnaire which includes several selfreport measures of psychiatric distress. At wave two, approximately twelve months following wave one, structured personal interviews are conducted to evaluate the psychiatric status of individual twins and their families. At the time of writing, coding and data entry of wave two data are still in progress.

Included in wave one are several measures of psychiatric symptomatology, life and role related stress, and suspected 'stress buffer' variables such as coping style, social support, and personal resources. Also included is the Center for Epidemiological Studies Depression Scale (CES-D) (Comstock \& Helsing, 1976; RadlofF, 1977); a 20-item questionnaire designed to measure the frequency of depressive symptomatology in the general population. Items used in the scale were taken from several measures of depression including those developed by Zung (1965), Beck, WARD, and MENDELson (1961), Raskin, Schulterbrandt, and Reatig (1967), and the depression subscale of the Minnesota Multiphasic Personality Inventory (Dahistrom \& Welsh, 1960). The CES-D has been shown to have adequate internal consistency across a range of samples (ROBERTs, 1980), and has been validated against other relevant measures of depressive symptoms (RADLOFF, 1977; Weissman, Sholomskas, Pottenger, Prusoff, \& Locke, 1977). Factor analytic studies of the CES-D has also demonstrated a comparable factor structure across a variety of race, sex, and ethnic groups (Clark, Aneshensel, Frerichs, \& Morgan, 1981; Aneshensel, Clark, \& Frerichs, 1983).

In the present study, twins were asked to respond to items on the CES-D scale on a four point scale based on the frequency of the particular symptom within the past week; i.e. (1) rarely or none of the time (less than 1 day), (2) some or little of the time (1-2 days), 
(3) occasionally or a moderate amount of the time (3-4 days), or (4) most of the time (5-7 days).

\section{Data summary and analysis}

Studies of twin pairs, unlike surveys of unrelated individuals, provide important checks on the adequacy of sampling. Truncated sampling, which can occur when very depressed twins do not respond, will lead to mean differences between twins from pairs where only one twin has responded, compared to pairs where both have responded. Further, if there are genetic effects on depressive symptoms, this will also lead to mean differences as a function of zygosity (Martin \& Wilson, 1982; Neale, Heath, Hewitt, Eaves, \& Fulker, 1989). To evaluate the representativness of our sample, we calculated the difference between the mean scores of depression for twins from complete and incomplete pairs, and the mean scores between the two zygosity groups.

Selecting an appropriate correlational statistic is a necessary first step in fitting factor models to twin data. Because the observations from the present study are based upon a discontinuous scale of measurement, it is not appropriate to use statistical methods which have been designed for the analysis of continuous variables. Using a Pearson correlation, for example, can lead to biased estimates and erroneous conclusions when analyzing discrete data (Olsson, 1979). Instead, we estimate polychoric correlations and polyserial correlations separately for each zygosity group by maximum likelihood (TALlis, 1962; Olsson, 1979; Olsson, Drasgow, \& Dorans, 1982). The polychoric correlation does not represent the correlation between the observed discontinous CES-D scores, but rather the correlation between two underlying normally distributed latent variables (e.g., the liabilities to depression of the two twins), which are assumed to be normally distributed. The estimation of the polychoric and polyserial correlations is based upon the assumption that the observed discontinuous measure(s) reflects the imposition of one or more 'thresholds' upon this underlying normal distribution of liability which can be tested by fitting the 'threshold' model to the analysis of twins' responses.

As a first step in formal data analysis, we performed a phenotypic factor analysis of the CES-D scale. We call it 'phenotypic' (another name for conventional factor analysis) to differentiate it from the factor models used in multivariate genetic analysis. This analysis allows an examination of the pattern of covariation of symptoms by reducing a large number of correlated variables to a smaller number of underlying latent common factors. It partitions the variation in item responses into 'common factor' variance, arising through the common influence of one or more latent factors on multiple items, and item-specific or residual variance. For our purposes we used the $20 \times 20$ correlation matrix of the responses of cotwins to each of the items of the CES-D scale from each zygosity group. We first performed a one factor principal factor analysis to determine whether a substantial proportion of the variation in the CES-D scale could be accounted for by a single depression factor. To permit comparability of the factor structure across different populations, we also performed a four factor phenotypic analysis. The latter was based upon previous analyses of the CES-D scale which have documented a four factor structure. In our analysis, the four factors were rotated to orthogonal simple structure by the varimax method (Harman, 1976). The congruency coefficient (Derogatis, Serio, \& Cleary, 1972) was 
also used to determine whether the factor loadings obtained from the two twin groups, i.e., twin 1 and twin 2, were similar enough to warrant combining them into one twin group.

In conventional factor analysis, we are primarily interested in the phenotypic correlation or covariation of individual items. Multivariate genetic analysis is a generalization of the more conventional factor analytic procedure in which we examine both the covariation among symptoms and also the genetical and environmental causes of this covariation. The clustering or patterning of symptoms can be explained by the influence of common and item-specific factors including additive genetic factors (VA), shared environmental factors (EC), and unique environmental factors (ES). Unique environmental influences can include error variance, and short term fluctuations, life events, and more long term effects specific to the individual.

Quantitative genetic theory predicts that the correlations between additive genetic factors will be 1.0 for monozygotic twin pairs, and 0.5 for dizygotic twins. For both zygosity groups, the correlation between the shared environmental factors will be 1.0. To estimate the loadings of symptoms on the latent factors, we analyze the correlations among items for both members of a twin pair (as in conventional factor analysis), and the cross correlations of items for twin 1 with items for twin 2, using the above predictions derived from genetic theory. By comparing the cross-twin, cross-item correlations between monozygotic and dizygotic twin pairs, (e.g., twin 1-item 1 with twin 2-item 2) we are able to estimate whether there are one or more common factors that account for the association between particular items and whether this association is genetically and/or environmentally determined.

To summarize the data for multivariate genetic analysis, we calculated a $41 \times 41$ matrix of correlations of the twins' age and responses of first and second twin to each of the 20 CES-D items, separately for MZ and DZ twin pairs. Twins were assigned as 'first' or 'second' on the basis of birth order, or at random if such information was unavailable. The twins' age was included as a way of controlling for its effect in the analysis of item responses (NEAlE \& MARTIN, 1989). Since both twins are the same age, this variable can spuriously inflate the estimate of the shared environment factor loadings if appropriate correction procedures are not employed.

After summarizing the data, the next step in multivariate genetic analysis is fitting models to the matrices of the polychoric and polyserial correlations. The method of maximum likelihood may be used for this purpose (c.f., Neale, Heath, Hewitt, Eaves, \& Fulker, 1989) but is limited by the requirement that the matrices of correlations be positive definite (JORESKOG \& SORBOM, 1988). When working with a large number of variables, as in the present case, this requirement often cannot be met, and other methods of model fitting must be used. Although these methods circumvent the problem of non positive definite matrices and provide estimates of the models' parameters, they are not able to provide a chi-squared significance test, and require us to rely on descriptive rather than statistical criteria for evaluating the fit of our models.

In the present study, two multivariate genetic models were fitted to the data by the method of unweighted least squarcs. In the first model, the 'latent phenotype model' (illustrated in Fig. 1), also referred to as the 'common pathway model' or 'psychometric model' (MCARDLE \& GoldSMith, 1984; KendLer et al., 1987), genes and environment are assumed to contribute to the same pattern of symptom covariation via one or more latent common 


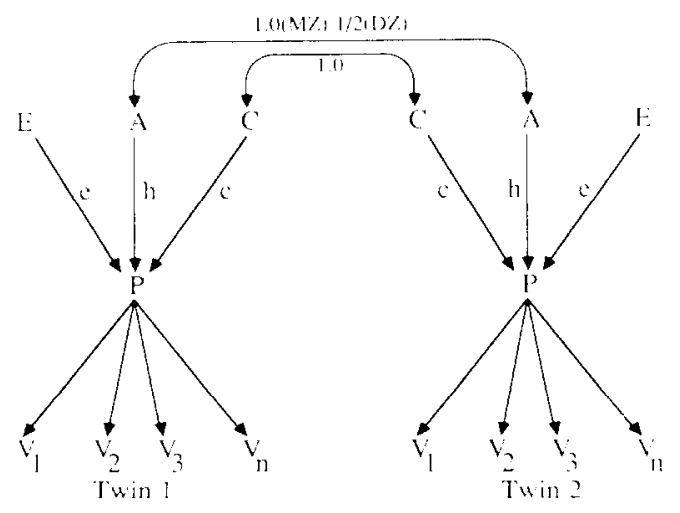

FIG. 1. Latent Phenotype Model

factors. Under this model, the genetic and environmental factor structures are assumed to be the same. Only the relative magnitudes of the three genetic and environmental parameters are estimable, by fixing one of the three parameters to unity and allowing the other parameters to vary (HEATH et al., 1989). The factor loadings on the common genetic and common shared environmental factors are estimated as constant multiples of loadings on the corresponding common unique environmental factor, fixing e $=1.0$. Standardized variance components of the latent phenotype are thereby derived as VA $=h^{2} /\left(h^{2}+e^{2}\right.$ $\left.\left.+c^{2}\right), \mathrm{EC}=c^{2} / h^{2}+e^{2}+c^{2}\right)$, and $\mathrm{ES}=e^{2} /\left(h^{2}+e^{2}+c^{2}\right)$, where $h^{2}+e^{2}+c^{2}$ is the overall variance of the latent phenotype.

One method of evaluating the fit of the latent phenotype model is to compare the similarity of item loadings on the latent common factor with those item loadings derived from a phenotypic factor analysis. If the model provides a good fit to the data, we would expect the loadings of items on a single underlying latent factor to correspond closely to item loadings on a single phenotypic factor. Similarly, in fitting a model with greater than one factor, the item loadings on each of the factors should compare to those loadings on the same number of factors derived from a phenotypic factor analysis. If the loadings from the multivariate model and the phenotypic model are not comparable we would conclude that the latent phenotype model cannot adequately account for the data observed.

In the more general version of the multivariate model, known as the "independent pathway model' (illustrated in Fig. 2), there is no assumption that genetic and environmental factors contribute to the covariation among symptoms in the same way. Rather, this model permits the detection of separate and distinct genetic and environmental influences on symptom covariation. It has been reported elsewhere that the covariation among the observed symptoms is influenced by relatively distinct genetic and environmental factor structures (Kendler et al., 1987; Silberg, Martin, \& Heath, 1987). In an analysis of personality traits, anxiety, depression, and menstrual symptomatology, SILBERG et al. (1987) reported that a common genetic factor could account for the covariation among menstrual symptoms, state anxiety, depression and the personality trait neuroticism. In contrast, environmental factors that affected the self-report of menstrual symptoms were not the same as those 


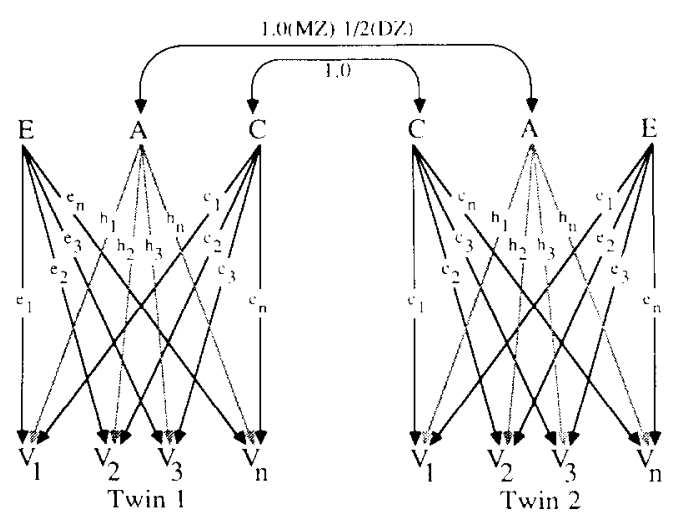

FIG. 2. Independent Pathway Model.

influencing the personality and distress variables. These findings point to separate genetic and environmental factor structures influencing the clustering of these variables, and implicate genetic effects in the correlations found between menstrual symptoms, personality, and psychiatric distress.

In fitting both the latent phenotype and independent pathway models, we begin with a one factor model as in the phenotypic factor analysis, and estimate additional common factors until the item specific loadings, those factors that influence one item of the scale but not any of the others, cannot be further reduced. To evaluate our models we also compare the similarity in factor loadings of the proposed model with those loadings derived from the phenotypic factor analysis.

In all models with more than one factor, the resulting factors are rotated to simple orthogonal structure by the varimax criterion, to ensure comparability with the phenotypic analysis of symptoms. Further, since we are primarily interested in the causal relationship among variables, we use an uncorrelated orthogonal rotation which provides a mathematically simple solution for interpreting the causes underlying symptom covariation.

\section{RESULTS}

The endorsement frequencies of each response category for the CES-D items are presented in Table 1. A large proportion of individuals report they had experienced a given symptom rarely or only a little of the time in the preceding 7 days, consistent with the use of the CES-D scale as an instrument for assessing psychopathology in the general population. Despite the predominance of low frequency symptoms, there does appear to be sufficient variability in the moderate and upper ranges of the distribution to permit a meaningful analysis of the scale.

To test the representativeness of our sample, we examined the extent to which a 'cooperation' factor may be restricting the range of depressive symptoms sampled. We found no significant difference between the mean scorcs for incomplete versus complete twin pairs ( $p=.88$ for $\mathrm{MZ}$ twins, $p=.35$ for $\mathrm{DZ}$ twins), or between the two zygosity 
Table 1. Endorsement Frequencies For the CES-D Scale Items

\begin{tabular}{|c|c|c|c|c|}
\hline \multirow{4}{*}{ CES-D Items } & \multicolumn{4}{|c|}{ Response categories } \\
\hline & 1 & 2 & 3 & 4 \\
\hline & \multicolumn{4}{|c|}{ Number of days* } \\
\hline & $<1$ & $1-2$ & $3-4$ & $5-7$ \\
\hline 1. I was bothered by things that don't usually bother me. & 1007 & 357 & 124 & 44 \\
\hline 2. I did not feel like eating; my appetite was poor. & 1064 & 297 & 126 & 49 \\
\hline 3. I felt that I could not shake off the blues, even with help & & & & \\
\hline from my family or friends. & 1044 & 288 & 127 & 61 \\
\hline 4. I felt that I was just as good as other people. & 164 & 199 & 313 & 833 \\
\hline 5. I had trouble keeping my mind on what I was doing. & 785 & 464 & 199 & 63 \\
\hline 6. I felt depressed. & 793 & 472 & 176 & 86 \\
\hline 7. I felt that everything I did was an effort. & 964 & 378 & 126 & 65 \\
\hline 8. I felt hopeful about the future. & 213 & 290 & 403 & 628 \\
\hline 9. I thought my life had been a failure. & 1303 & 161 & 44 & 20 \\
\hline 10. I felt fearful. & 1293 & 173 & 44 & 22 \\
\hline 11. My sleep was restless. & 727 & 459 & 234 & 111 \\
\hline 12. I was happy. & 94 & 217 & 440 & 772 \\
\hline 13. I talked less than usual. & 790 & 488 & 198 & 51 \\
\hline 14. I felt lonely. & 986 & 339 & 123 & 86 \\
\hline 15. People were unfriendly. & 1208 & 248 & 54 & 24 \\
\hline 16. I enjoyed life. & 79 & 207 & 409 & 837 \\
\hline 17. I had crying spells. & 973 & 225 & 89 & 40 \\
\hline 18. I felt sad. & 880 & 467 & 131 & 55 \\
\hline 19. I felt that people dislike me. & 1215 & 237 & 48 & 30 \\
\hline 20. I could not get going. & 812 & 507 & 155 & 61 \\
\hline
\end{tabular}

*See text for exact wording of item frequency.

groups $(p=.09)$. Thus we concluded that any effect of differential responding by the twins was not substantially truncating the range of symptoms in our sample.

As discussed previously, the estimation of polychoric and polyserial correlations rests upon the assumption that the discontinuous CES-D scores represent thresholds that are superimposed upon an underlying normal distribution of liability. To test the validity of this assumption, we fitted the threshold model to the twins' univariate item responses, and found that this model provided a good fit to the twins' responses to the 20 CES-D items, justifying the use of the polychoric and polyserial correlations in our analyses.

\section{Phenotypic factor analysis}

The congruency coefficient, an index of the similarity between factor loadings for twin 1 and twin 2, was .98. Given the strong agreement between factor structures, we combined the two twin groups for all subsequent analyses.

The results of a one factor and four factor phenotypic analysis of the CES-D scale are presented in Table 2. We factor analyzed the CES-D with a one factor principal factor analysis to determine whether a single 'depression' factor could account for a large proportion of variance in the scale. This unitary factor accounts for an average of $31.3 \%$ of the variance in the CES-D items, and has its highest loadings on those items that are primarily mood related (i.e., items $3,6,12,14$, and 18) and relatively weaker loadings on those items reflecting appetite disturbance (2), low self-esteem (4), hopelessness (8), and interpersonal sensitivity (15). 
Table 2. Phenotypic Factor Loadings (X 100) for the CES-D Scale: One Factor and Four Factor Solutions

\begin{tabular}{|c|c|c|c|c|c|}
\hline \multirow[t]{2}{*}{ Item } & \multirow{2}{*}{$\begin{array}{c}\text { One factor } \\
\text { I }\end{array}$} & \multicolumn{4}{|c|}{ Four factor } \\
\hline & & $\mathbf{I}$ & II & III & IV \\
\hline 1. I was bothered by things that don't usually bother me. & 50 & 31 & 39 & -10 & 12 \\
\hline 2. I did not fecl like eating; my appetite was poor. & 34 & 20 & 34 & -5 & 3 \\
\hline 3. I felt that I could not shake off the blues, even with help from & & & & & \\
\hline my family or friends. & 76 & 59 & 38 & -29 & 15 \\
\hline 4. I felt that I was just as good as other people. & -33 & -9 & -2 & 50 & -13 \\
\hline 5. I had trouble keeping my mind on what I was doing. & 52 & 18 & 57 & -10 & 17 \\
\hline 6. I felt depressed. & 52 & 62 & 41 & -32 & -13 \\
\hline 7. I felt that everything I did was an effort. & 57 & 20 & 57 & -16 & 16 \\
\hline 8. I felt hopeful about the future. & -38 & -12 & -6 & 54 & -8 \\
\hline 9. I thought my life had been a failure. & 56 & 33 & 22 & -29 & 32 \\
\hline 10. I felt fearful. & 46 & 29 & 31 & -11 & 16 \\
\hline 11. My sleep was restless. & 44 & 22 & 43 & -10 & -6 \\
\hline 12. I was happy. & -65 & -23 & -31 & 72 & -8 \\
\hline 13. I talked less than usual. & 42 & 27 & 30 & -9 & 11 \\
\hline 14. I felt lonely. & 68 & 54 & 31 & -25 & 21 \\
\hline 15. People were unfriendly. & 39 & 11 & 21 & -13 & 46 \\
\hline 16. I enjoyed life. & -59 & -18 & -27 & 70 & -9 \\
\hline 17. I had crying spells. & 58 & 61 & 25 & -11 & 11 \\
\hline 18. I felt sad. & 77 & 74 & 28 & -25 & 20 \\
\hline 19. I felt that people dislike me. & 52 & 22 & 15 & -16 & 80 \\
\hline 20. I could not get going. & 57 & 16 & 57 & -22 & 17 \\
\hline
\end{tabular}

T'o replicate the four factor phenotypic solution previously reported by RADLOFF (1977), we also factor analyzed the CES-D scale with a four factor principal factor analysis. In both analyses, factor one is interpretable as a 'depressed or negative affect' factor which has high loadings $(>0.4)$ on the following items: "I felt I could not shake off the blues, even with help from family or friends", "I felt depressed", "I felt lonely", "I had crying spells", and "I felt sad". Factor two, a somatization, poor concentration and motivation factor has loadings on the items: "I had trouble keeping my mind on what I was doing", "I felt everything I did was an effort", "My sleep was restless", and "I could not get going". Factor three is a 'positive affect factor' and is defined by the following: "I felt that I was just as good as other people", "I felt hopeful about the future", "I was happy", and "I enjoyed life". Finally, the fourth factor is an "interpersonal sensitivity factor" with loadings on only two items: "People were unfriendly" and "I felt that people dislike me". In this factor solution, the four factors together account for an average of $42.4 \%$ of the phenotypic variance of the 20 item scale.

\section{Multivariate genetic analysis}

Fitting the latent phenotype model. The common factor loadings and estimates of the standardized separate genetic and environmental components of variance for the one factor and four factor latent phenotype models are presented in Table 3. The item-specific genetic and environmental factor loadings and the age correction factor loadings (which are essentially nuisance parameters) are not tabulated. We selected the one factor and four factor solutions as ways of comparing the factor structure derived from the multivariate analysis to the phenotypic structure described previously. 
Table 3. Parameter Estimates and Common Factor Loadings (X 100) for the One Factor and Four factor latent Phenotype Models

\begin{tabular}{lrrrrr}
\hline \multicolumn{1}{c}{ Item } & One factor & \multicolumn{3}{c}{ Four factor } \\
& I & I & II & III & IV \\
\hline VA & 29 & 55 & 33 & 00 & 00 \\
EC & 13 & 00 & 00 & 26 & 38 \\
ES & 58 & 45 & 67 & 74 & 62 \\
\hline 1. I was bothered by things. & 39 & 19 & 5 & 29 & 26 \\
2. I did not feel like eating. & 31 & 8 & 9 & 26 & 26 \\
3. I could not shake off the blues. & 64 & 32 & 22 & 51 & 25 \\
4. I felt as good as others. & -34 & -22 & -44 & -2 & -1 \\
5. I had trouble concentrating. & 41 & 16 & 7 & 22 & 43 \\
6. I felt depressed. & 65 & 32 & 23 & 52 & 26 \\
7. I felt everything I did was an effort. & 49 & 23 & 10 & 26 & 43 \\
8. I felt hopeful about the future. & -36 & -19 & -51 & 2 & -8 \\
9. I thought my life had been a failure. & 58 & 35 & 27 & 26 & 24 \\
10. I felt fearful. & 43 & 23 & 10 & 29 & 25 \\
11. My sleep was restless. & 37 & 17 & 4 & 23 & 32 \\
12. I was happy. & -57 & -26 & -54 & -16 & -26 \\
13. I talkcd lcss than usual. & 34 & 17 & 12 & 23 & 18 \\
14. I felt lonely. & 58 & 37 & 19 & 39 & 16 \\
15. People were unfriendly. & 37 & 43 & 1 & -9 & 23 \\
16. I enjoyed life. & -53 & -26 & -51 & -13 & -22 \\
17. I had crying spells. & 54 & 31 & 15 & 54 & 7 \\
18. I felt sad. & 64 & 41 & 19 & 54 & 10 \\
19. I felt that people dislike me. & 51 & 56 & -1 & 8 & 19 \\
20. I could not get going. & 49 & 25 & 13 & 19 & 44 \\
\hline
\end{tabular}

The factor loadings under the one factor latent phenotype model are comparable with those loadings derived from the one factor phenotypic analysis. A single 'depression' factor accounts for an average of $23.9 \%$ of the variance in the scale's items. Under this model, additive genetic variance explains $29 \%$ of the variance in the latent phenotype (this is the heritability of the latent 'depression' phenotype), shared environmental effects $13 \%$, and unique environmental effects that make one twin differ from her cotwin, the remaining $58 \%$.

As a supplement to Table 3, we also present the results of the four factor common pathway model for the first two CES-D items in Fig. 3. Under the four factor model, which

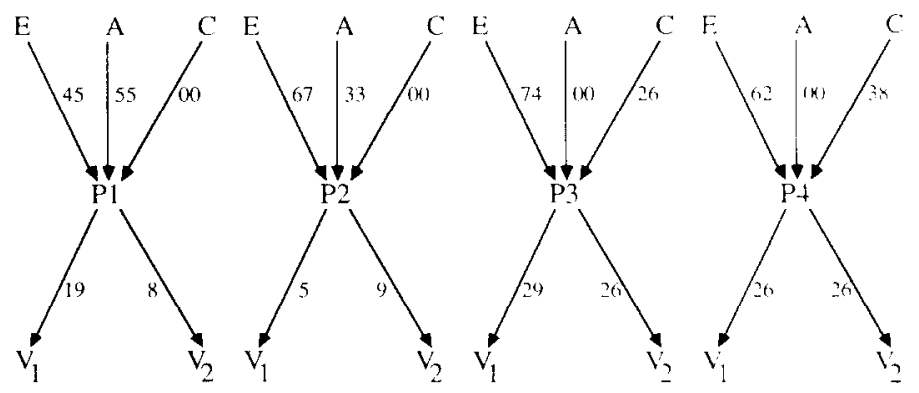

FIG. 3. Results of Fitting the Four Factor Latent Phenotype Model (CES-D items 1 and 2). 
assumes four underlying latent factors, the factor loadings vary considerably from those of the four factor phenotypic analysis. For two of the four factors, factor III, a general depression factor, and factor IV, a somatization, poor concentration and motivation factor, the heritability is zero and shared environmental influences account for $26 \%$ and $38 \%$ of the variance, respectively. For factor I, a depressed affect and interpersonal sensitivity factor, and factor II, a positive affect factor, this finding is reversed; the shared environment accounts for $0 \%$ of the variance in the two latent phenotypes whereas additive genetic variance explains $55 \%$ and $33 \%$, respectively. These results strongly suggest that separate genetic and environmental factor structures may be acting upon symptom covariation in different ways.

Fitting the independent pathway model. From the twin correlations and cross-twin crossitem correlations upon which the multivariate genetic analysis was based, it was apparent that a significant proportion of the variance in responses to the scale is due to non-shared environmental influences. We therefore selected an independent pathway model that estimated one genetic, one shared environmental factor, and four distinct non-shared environmental factors. Elaborations on this model were made by the addition of up to three genetic and three shared environmental factors. However, these additional factors had relatively low loadings on all but a few of the items, suggesting the need to estimate only one common genetic factor and one shared environmental factor to explain crosstwin symptom covariation. The factor loadings of items under the I-I-IV independent pathway model are presented in Table 4.

Table 4. Common Factor Loadings for the Independent Pathway Model (X 100)

\begin{tabular}{|c|c|c|c|c|c|c|c|c|c|}
\hline \multirow[t]{2}{*}{ Item } & \multicolumn{2}{|c|}{ Genetic } & \multicolumn{2}{|c|}{ Shared ENV } & \multicolumn{5}{|c|}{ Unique ENV } \\
\hline & I & SP & I & SP & $\mathbf{I}$ & II & III & IV & SP \\
\hline 1. I was bothered by things. & 22 & $\infty 0$ & 30 & 17 & 26 & 29 & -07 & 07 & 82 \\
\hline 2. I did not feel like eating. & 21 & 00 & 12 & 41 & 14 & 46 & -04 & -03 & 71 \\
\hline 3. I could not shake off the blues. & 39 & 02 & 34 & 07 & 57 & 32 & -23 & 09 & 48 \\
\hline 4. I felt as good as others. & -46 & 16 & 10 & 00 & -07 & -04 & 45 & 07 & 73 \\
\hline 5. I had trouble concentrating. & 15 & 07 & 31 & 31 & 17 & 48 & -14 & 17 & 67 \\
\hline 6. I felt depressed. & 40 & 04 & 39 & 00 & 57 & 31 & -24 & 04 & 45 \\
\hline 7. I felt everything I did was an effort. & 23 & 17 & 36 & 00 & 23 & 46 & -16 & 17 & 67 \\
\hline 8. I felt hopeful about the future. & -40 & 38 & 03 & 00 & -04 & -08 & 54 & -06 & 62 \\
\hline 9. I thought my life had been a failure. & 33 & 00 & 36 & 23 & 38 & 15 & -40 & 24 & 57 \\
\hline 10. I felt fearful. & 25 & 35 & 25 & 00 & 30 & 31 & -14 & 12 & 73 \\
\hline 11. My sleep was restless. & 21 & 21 & 31 & $\infty$ & 14 & 40 & -06 & 05 & 79 \\
\hline 12. I was happy. & -44 & 00 & -25 & 00 & -19 & -21 & 65 & -01 & 49 \\
\hline 13. I talked less than usual. & 22 & 16 & 03 & 09 & 26 & 35 & -11 & 13 & 83 \\
\hline 14. I felt lonely. & 43 & 24 & 23 & 00 & 49 & 24 & -19 & 16 & 57 \\
\hline 15. People were unfriendly. & 37 & 28 & 20 & 00 & 00 & 18 & -09 & 52 & 62 \\
\hline 16. I enjoyed life. & -38 & 00 & -19 & $\infty$ & -21 & -18 & 65 & -08 & 56 \\
\hline 17. I had crying spells. & 44 & 00 & 15 & 35 & 63 & 25 & -05 & 03 & 45 \\
\hline 18. I felt sad. & 52 & 00 & 27 & 09 & 66 & 18 & -12 & 12 & 38 \\
\hline 19. I felt that people dislike me. & 37 & 39 & 27 & 00 & 33 & 10 & -11 & 68 & 00 \\
\hline 20. I could not get going. & 28 & 29 & 36 & 00 & 15 & 44 & -19 & 18 & 64 \\
\hline
\end{tabular}


As can be seen from this table, the item loadings under the independent pathway model are smaller than those derived from the phenotypic analysis. This is to be expected since we are now partitioning the overall phenotypic variance into one genetic, one shared environmental, and four unique environmental factors.

The unique environmental factor loadings are quite similar to those obtained under the four factor phenotypic analysis described above. That is, factor I is characterized by items that load highly on 'depression', factor II on 'positive affect', factor III on somatic items, and factor IV on interpersonal sensitivity. Together these factors account for an average of $35 \%$ of the variance in the scale. The item-specific unique environmental variance, i.e., non-shared environmental variance affecting only one item of the scale, but not any other, is substantial.

The single common genetic factor accounts for $12.9 \%$ of the variance in the CES-D items, and loads weakly on the somatic-poor motivation, and concentration items. It has high (positive) loadings on those items reflecting depressed affect and low self-esteem, loneliness, and interpersonal sensitivity, in addition to high (negative) loadings on positive affect. The item-specific genetic loadings (genetic variance specifically affecting only one item on the scalc) are rclativcly small. For most of the items the single common genctic factor accounts for most of the genetic variance in the twins' responses. The common shared environmental factor loads significantly on a variety of depressive and somatic, poor concentration and motivation symptoms, but in contrast to the common genetic factor, loads relatively weakly on those items measuring positive affect. This common shared environmental factor accounts for only $5 \%$ of the variation in the CES-D scale. Many of the item-specific genetic or shared environmental loadings have been estimated as zero. This probably reflects the lack of precision with which these residual effects are estimated.

\section{DISCUSSION}

To examine the causes of covariation in self-reported depressive symptoms, we fitted multivariate genetic models to the responses of 771 pairs of twins to the 20 items of the CES-D scale (RADLOFF, 1977). Although the fit of these models could not be statistically evaluated, an independent pathway model allowing for one common genetic factor, one shared environmental factor, and four unique environmental factors provided a particularly useful description of how depressive symptoms co-occur in the general population. Under this model, the four common non-shared environmental factors (reflecting the influence of environmental events specific to the individual) accounted for the largest proportion of variance in the report of symptoms. The item loadings on these four factors were similar to those obtained from a phenotypic factor analysis of the CES-D scale, and included a 'depressed affect' factor, a 'positive affect' factor, a 'somatization' factor and a factor measuring 'interpersonal sensitivity'. The strong correspondence between these two models suggests that most of the covariation of symptoms observed in the phenotypic factor analysis is primarily due to non-shared environmental influences.

The importance of unique environmental factors in symptoms of anxiety and depression has been previously demonstrated (KENDLER et al., 1986, 1987), and is consistent with theories positing the importance of adult life experiences in symptoms of psychiatric distress 
(PAYKel, 1982). In an analysis of the Delusions-Symptoms Inventory (BEDFORD, Foulds, \& SHefFIELD, 1976) KENDLER et al. (1987) reported that three common non-shared environmental factors accounted for $34.3 \%$ of the variance in the anxiety and depressive items comprising this scale. This is similar in magnitude to our estimate of $35 \%$ of the variance for the depressive items of the CES-D.

In our study, genetic effects have also been shown to influence the covariation among depressive items, specifically symptoms of depressed affect, low self-esteem, loneliness, and interpersonal sensitivity. However, only $12.9 \%$ of the variation in depressive symptoms could be accounted for by a single genetic factor, an estimate considerably lower than the $27 \%$ reported by KENDLER et al. (1987) for the first common genetic factor influencing symptoms of anxiety and depression in an Australian twin sample. It might be that crosscultural differences in the variance of environmental determinants explains this discrepancy, although incomparability between the inventories used in the two studies makes it impossible to test this assumption directly.

Interestingly, in the analysis of the Delusions-Symptoms Inventory, a model containing three genetic and three non-shared environmental factors provided a good fit to items reflecting sleep disturbance and low motivation and energy. In contrast, in the present study, sleep and appetite disturbance, and poor motivation and concentration, were found to have minimal loadings on the common genetic factor as well as small item-specific genetic loadings, demonstrating the importance of environmental rather than genetic effects in the etiology of these particular symptoms.

This inconsistency in genetic effects on individual symptoms of psychiatric distress may be due to the use of different populations upon which the estimates are based. Further, differences in the proportion of variance accounted by a common genetic factor may be explained by the use of different instruments for measuring symptoms. The DelusionsSymptoms Inventory samples a smaller range of behaviors, and includes anxiety as well as depressive items. In addition, the depressive symptoms measured represent slightly different aspects of depressive behavior than those included in the CES-D scale. Given the inconsistencies between these two studies, a definitive statement regarding the extent of genetic effects on depressive symptoms will necessarily require further investigation.

A growing trend emerging from the field of behavior genetics is the negligible effect of the common family environment on symptoms of psychiatric distress (Plomin \& DANIELS, 1987). Consistent with these findings, the shared family environmental component accounted for a relatively small portion of the overall environmental variance in depressive symptoms (i.e., 5\%). Although the evidence suggests that sharing a common rearing environment does not play an important causal role in the onset of self-reported depressive symptoms, it is possible that family dynamics are not similarly influencing all members of the same family, or that socialization experiences that are important for the development of adult depression vary between twins in the same family. Before ruling out the importance of these factors in the etiology of symptoms, one must address the important question as to the extent to which family dynamics may be specifically effecting only one member of a twin pair.

Several limitations of the present study deserve mention. The most obvious shortcoming is the lack of statistical rigor in evaluating our models. Given the large number of 
discontinuous variables included in the analysis, we are forced to rely on descriptive rather than statistical criteria in determining how well our models fit the data.

Onc advantage of the twin study method is that by comparing the monozygotic twin correlations to the test-retest correlations, one is able to partition the overall non-shared environmental variance into measurement error (and/or short term fluctuations in behavior), and more long term environmental effects. Since test-retest data are unavailable at this time, these components of environmental variance cannot be fully delineated, and we must therefore be somewhat circumspect in our conclusions about genetic and environmental effects. If the CES-D is an unreliable measure, for example, the estimates of genetic and common environmental factors for the self-report of depressive symptoms could be spuriously lowered. However, given the strong correspondence between the multivariate environmental structure and the factor stucture derived from the phenotypic analysis, it is unlikely that the non-shared environmental variance reflects only measurement error.

The twin method has also been criticized on the grounds that twins share a 'special twin environment'. Specifically, the use of twins can lead to inflated estimates of additive genetic variance if the greater similarity between monozygotic twins in comparison to dizygotic twin pairs reflects the sharing of a special environment rather than a more similar genetic make up. To test this, we separately examined the association between the frequency of intrapair contact during childhood and adulthood, and similarity in depressive symptoms currently reported. Since our data showed no significant association between the amount of time twins spent together as children and similarity in their symptoms, or their current level of contact and present symptomatology, we found no evidence for a 'special twin environment' that could limit the generalizability of our findings to a non-twin population.

Finally, because the findings from the present study are based on self-reported depressive symptoms they should not be generalized to formal psychiatric syndromes nor assumed to reflect DSM-III-R criteria for Depressive Disorders (American Psychiatric Association, 1987).

If, however, self-reported depressive symptoms are found to be associated with depressive syndromes, the finding of separate genetic and phenotypic factor structures may suggest different familial versus non-familial patterns of symptoms, and be used to identify particular individuals at risk. For example, LECKMAN et al. (1984) reported evidence of increased risk of major depression in relatives of depressed probands with symptoms of appetite disturbance and excessive guilt. Similarly, those depressive symptoms in the present study that were found to be genetically determined may serve as predictors of risk in the family members of those individuals reporting these symptoms.

The present study represents only one portion of a larger study of symptoms of anxiety and depression in Virginia twins. Although we have examined the importance of genetic and environmental factors in these symptoms, we have not yet specified those environmental events that may be related to their onset. Further, the role of genes and the environment in psychiatric syndromes of anxiety and depression in this population remains to be explored. To address these questions, we have collected data from other self-report measures of psychiatric distress (e.g., Hopkins, Symptoms Checklist) and data from clinical interviews with twins and their families. Assuming an important role of genes in psychiatric symptoms, this data can provide us with a model that will help us identify specific factors that may 
alter the relationship between genes and the expression of symptoms of distress, and may help to clarify the relationship between depressive symptoms and clinically relevant syndromes.

Acknowledgements-This work was made possible through NIH Grants MH-40828 amd AG-04954. The authors are grateful for helpful comments from Marilyn T. Erickson, John K. Hewitt and two anonymous reviewers of the manuscript.

\section{REFERENCES}

American Psychiatric Association (1987). Diagnostic and statistical manual of mental disorders (3rd ed.). Washington, DC: American Psychiatric Association.

Aneshensel, C., Clark, V., \& Frerichs, R. (1983). Race, ethnicity, and depression: A confirmatory analysis. Juurnal of Personulity and Social Psychology 44, 385-398.

BECK, A. T., WARD, C. H., \& MENDELSON, M. (1961). An inventory for measuring depression. Archives of General Psychiatry 4, 561-571.

Bedford, A., Foulds, G. A., \& Sheffield, B. F. (1976). A new personal disturbance scale (DSSI/SAD). British Journal of Social and Clinical Psychiatry 15, 387-394.

Bertelsen, A., Harvald, B., \& Hauge, M. (1977). A Danish twin study of manic-depressive disorders. British Journal of Psychiatry 130, 330-351.

Brown, G. W., Harris, T. O., \& Copeland, J. R. (1977). Depression and loss. British Journal of Psychiatry 130, $1-18$.

Cadoret, R. J. (1978). Evidence for genetic inheritance of primary affective disorder in adoptees. American Journal of Psychiatry 135, 463-466.

Cadoret, R. J., O'Gorman, T. W., Heywood, E., \& Troughton, E. (1985). Genetic and environmental factors in major depression. Journal of Affective Disorders 9, 155-164.

Clark, V. A., Aneshensel, C. S., Frerichs, R. R., \& Morgan, T. M. (1981). Analysis of effects of sex and age in response to items on the CES-D scale. Psychiatry Research 5, 171-181.

Comstock, G. W. \& Helsing, K. (1976). Symptoms of depression in two communities. Psychological Medicine 6, 551-563.

Corey, L. A., Eaves, L. J., Mellen, B. G., \& Nance, W. E. (1986). Testing for developmental changes in gene expression on resemblance for quantitative traits in kinships of twins: application to height, weight, and blood pressure. Cenetic Epidemiology 3, 73-83.

Dahlstrom, W. G. \& Welsh, G. S. (1960). An MMPI handbook. Minneapolis: University of Minnesota Press.

Derogatis, L. R., Serio, J. C., \& Cleary, P. A. (1972). An empirical comparison of three indices of factorial similarity. Psychological Report 30, 791-804.

Gershon, E. S., Bunney, W. E., Jr., Leckman, J. F., van Eerdewegh, M., \& DeBauche, B. A. (1976). The inheritance of affective disorders: a review of data and of hypotheses. Behavior Genetics 6, 227-261.

Harman, H. H. (1976). Modern factor analysis. Chicago: University of Chicago Press.

Heath, A. C., Neale, M. C., Hewitt, J. K., Eaves, L. J., \& Fulker, D. W. (1989). Testing structural equation models for twin data using LISREL. Behavior Genetics 19, 9-35.

Jardine, R., Martin, N. G., \& Henderson, A. S. (1984). Genetic covariation between neuroticism and the symptoms of anxiety and depression. Genetic Epidemiology 1, 89-107.

JORESKOG, K. G. \& SORBOM, D. (1988). Lisrel VII: a guide to the program and its applications. Chicago, Illinois: SPSS.

Kendler, K. S., Heath, A. C., Martin, N. G. \& Eaves, L. J. (1986). Symptoms of anxiety and depression in a volunteer twin population. Archives of General Psychiatry 43, 213-221.

Kendler, K. S., Heath, A. C., Martin, N. G., \& Eaves, L. J. (1987). Symptoms of anxiety and depression: Same genes, different environments? Archives of General Psychiatry 44, 451-457.

KETY, S. S. (1985). Interactions between stress and genetic processes. In M. R. Zales (Ed.), Stress in Health and Disease. New York: Brunner/Mazel.

Leckman, J. F., Caruso, K. A., Prusoff, B. A., Weissman, M. W., Merikangas, K., \& Pauls, D. L. (1984). Appetite disturbance and excessive guilt in major depression. Archives of General Psychiatry 41, 839-844.

Martin, N. G. \& Eaves, L. J. (1977). The genetical analysis of covariance structures. Heredity 38, 79-95.

MARTIN, N. G. \& Wilson, S. R. (1982). Bias in the estimation of heritability from truncated samples of twins. Behavior Genetics 12, 467-472. 
MCARdie, J. \& Goldsmith, H. H. (1984). Structural equation modeling applied to the twin design: Comparative multivariate models of the WAIS. Behavior Genetics 14, 609.

Mendlewicz, J. \& Rainer, J. D. (1977). Adoption study supporting genetic transmission in manic-depressive illness. Nature 268, 327-329.

Neale, M. C., Heath, A. C., Hewitt, J. K., Eaves, L. J., \& Fulker, D. W. (1989). Fitting genetic models with LISREL: hypothesis testing. Behavior Genetics 19, 37-49.

Neale, M. C. \& MarTin, N. G. (1989). The effects of agc, sex, and genotype on sclf-rcport drunkenness following a challenge dose of alcohol. Behavior Genetics 19, 63-78.

Nichols, R. C. \& Bilbro, W. C. (1966). The diagnosis of twin zygosity. Acta 16, 265-275.

OLsson, U. (1979). Maximum likelihood estimation of the polychoric correlation coefficient. Psychametrika 44, 433-460.

Olsson, U., Drasgow, F., \& Dorans, N. J. (1982). The polyserial correlation coefficient. Psychometrika 47, 337-347.

PARKer, G. (1979). Parental characteristics in relation to depressive disorders. British Journal of Psychiatry 134, $138-147$.

PAYKel, E. S. (1982). Life events and early environment. In E. S. Paykel (Ed.), Handbook of affective disorders. New York: Guilford Press.

Plomin, R. \& Daniels, D. (1987). Why are children in the same family so different from one another? Behavioral and Brain Sciences 10, 1-60.

RADLOFF, L. S. (1977). The CES-D scale: A self-report depression scale for research in the general population. Applied Psychological Measurement 1, 385-401.

Raskin, A., Schulterbrandt, J., \& Reatig, N. (1967). Factors of psychopathology in interview, ward behavior, and self-report of hospitalized depressives. Juurnal of Consulting Psychology 31, 270-278.

RoBerts, R. E. (1980). Reliability of the CES-D scale in different ethnic contexts. Psychiatry Research 2, $125-134$.

Silberg, J. L., Martin, N. G., \& Heath, A. C. (1987). Genetic and environmental factors in primary dysmenorrhea and its relationship to anxiety, depression, and neuroticism. Behavior Genetics 17, 363-383.

Tallis, G. M. (1962). The maximum likelihood estimation of correlation from contingency tables. Biometrics $18,342-353$.

Torgersen, S. (1986). Genetic factors in moderately severe and mild affective disorders. Archives af General Psychiatry 43, 222-226.

Von Knorring, A.-L., Cloninger, R., Bohman, M., \& Sigvardson, S. (1983). An adoption study of depressive disorders and substance abuse. Archives of General Psychiatry 40, 943-950.

Weissman, M., Sholomskas, D., Pottenger, M., Prusoff, B., \& Locke, B. Z. (1977). Assessing depressive symptoms in five psychiatric populations: A validation study. American Journal of Epidemiology 106, 203-214.

Wender, P. H., Kety, S. S., Rosenthal, D., Schuslinger, F., Ortmann, J., \& Lunde, I. (1986). Psychiatric disorders in the biological and adoptive relatives of adopted individuals with affective disorders. Archives of General Psychiatry 43, 923-929.

WinOKuR, G. \& Clayton, P. (1967). Family history study I: Two types of affective disorder separated according to genetic and clinical factors. In J. Wortis (Ed.). Advances In Biological Psychiatry 9, 35-50. New York: Plenum Press.

Zung, W. W. K. (1965). A self-rating depression scale. Archives of General Psychiatry 12, 63-70. 